\title{
Chiral symmetry breaking and the spin content of hadrons
}

\author{
L. Ya. Glozman, C. B. Lang, M. Limmer \\ Institut für Physik, FB Theoretische Physik, Universität Graz, Universitätsplatz 5, \\ A-8010 Graz, Austria
}

October 19, 2018

\begin{abstract}
From the parton distributions in the infinite momentum frame one finds that only about $30 \%$ of the nucleon spin is carried by spins of the valence quarks, which gave rise to the term "spin crisis". Similar results hold for the lowest mesons, as it follows from the lattice simulations. We define the spin content of a meson in the rest frame and use a complete and orthogonal $\bar{q} q$ chiral basis and a unitary transformation from the chiral basis to the ${ }^{2 S+1} L_{J}$ basis. Then, given a mixture of different allowed chiral representations in the meson wave function at a given resolution scale, one can obtain its spin content at this scale. To obtain the mixture of the chiral representations in the meson we measure in dynamical lattice simulations a ratio of couplings of interpolarors with different chiral structure. For the $\rho$ meson we obtain practically the ${ }^{3} S_{1}$ state with no trace of the spin crisis. Then a natural question arises: which definition does reflect the spin content of a hadron?
\end{abstract}

\section{Introduction}

The nucleon spin structure function $g_{1}$, extracted from the deep inelastic lepton-nucleon scattering with polarization 1, 2], if interpreted in the infinite momentum frame, defines a fraction of the nucleon spin carried by quark spins. Accordingly, only about $30 \%$ of the nucleon spin is carried by quark spins, which is referred to usually as "spin crisis". Consequently, a lot of experimental and theoretical efforts for the last 20 years were devoted to search for the rest of the nucleon spin, without obvious success, however. Similar results are obtained on the lattice both for nucleons and lowest mesons (for a review see ref. [3]).

In [4, 5, 6] we have suggested a way to define in a gauge invariant manner and measure the spin content of mesons in the rest frame at different resolution scales, including the infrared ones. The method is based on the unitary transformation from the complete and orthogonal chiral quark-antiquark basis to the complete and orthogonal ${ }^{2 S+1} L_{J}$ basis in the rest frame [7]. Using a set of interpolators that transform according to different chiral representations [8, 9], one can measure on the lattice a ratio of couplings of different interpolators to a given state in the rest frame. This ratio determines a mixture of different chiral representations in the meson wave functions. Then, given this ratio and using a unitary transformation from the chiral basis to the angular momentum basis, one obtains the angular momentum content of a meson in the rest frame. 
The result was that the angular momentum content of the $\rho$-meson in the rest frame is given approximately by the ${ }^{3} S_{1}$ wave, without obvious trace of the spin crisis. Then a natural question is which of the definitions does reflect the spin content of a hadron?

Below we overview both principal aspects of the method as well as the numerical results using as an example the $\rho$-meson.

\section{The method}

The $I=1, J^{P C}=1^{--} \bar{q} q$ states with unbroken chiral symmetry transform according to two chiral representations, $(0,1)+(1,0)$ and $(1 / 2,1 / 2)_{b}[8,9]$. These two representations form a complete and orthogonal basis. The state that transforms as $(0,1)+(1,0)$ can be created from the vacuum by the vector current,

$$
O_{\rho}^{V}(x)=\bar{q}(x) \gamma^{i} \vec{\tau} q(x)
$$

and the state that belongs to the $(1 / 2,1 / 2)_{b}$ representation can be created by the pseudotensor operator,

$$
O_{\rho}^{T}(x)=\bar{q}(x) \sigma^{0 i} \vec{\tau} q(x) .
$$

In the continuum the physical $\rho$-meson with broken chiral symmetry can be created from the vacuum by both operators and the corresponding amplitudes are given as

$$
\begin{aligned}
\left\langle 0\left|\bar{q}(0) \gamma^{\mu} q(0)\right| V(p ; \lambda)\right\rangle & =m_{\rho} f_{\rho}^{V} e_{\lambda}^{\mu}, \\
\left\langle 0\left|\left(\bar{q}(0) \sigma^{\alpha \beta} q(0)\right)(\mu)\right| V(p ; \lambda)\right\rangle & =\mathrm{i} f_{\rho}^{T}(\mu)\left(e_{\lambda}^{\alpha} p^{\beta}-e_{\lambda}^{\beta} p^{\alpha}\right),
\end{aligned}
$$

where $V(p ; \lambda)$ is the vector meson state with the mass $m_{\rho}$, momentum $p$ and polarization $\lambda$. The vector current is conserved, consequently the vector coupling constant $f_{\rho}^{V}$ is scale-independent. The pseudotensor "current" is not conserved and is subject to a nonzero anomalous dimension. Consequently the pseudotensor coupling $f_{\rho}^{T}(\mu)$ manifestly depends on the resolution scale $\mu$. The very fact that the $\rho$-meson can be created by the operators that transform according two different chiral representations tells that chiral symmetry is broken and the meson wave function is a superposition of both chiral representations.

In the rest frame the ratio

$$
\frac{f_{\rho}^{V}}{f_{\rho}^{T}(\mu)}=\frac{\left\langle 0\left|\bar{q}(0) \gamma^{i} q(0)\right| V(\lambda)\right\rangle}{\left\langle 0\left|\left(\bar{q}(0) \sigma^{0 i} q(0)\right)(\mu)\right| V(\lambda)\right\rangle}
$$

determines the ratio of the two allowed chiral representations in the $\rho$-meson wave function at a given resolution scale $\mu$. Such ratio can be measured on the lattice.

Given a set of operators $O_{i}$ above we use the variational method [10] and calculate a cross-correlation matrix at zero spatial momentum (i.e., in the rest frame),

$$
C(t)_{i j}=\left\langle O_{i}(t) O_{j}^{\dagger}(0)\right\rangle=\sum_{n=1}^{\infty} a_{i}^{(n)} a_{j}^{(n) *} \mathrm{e}^{-E^{(n)} t},
$$

with the coefficients giving the overlap of the operators with the physical state,

$$
a_{i}^{(n)}=\left\langle 0\left|O_{i}\right| n\right\rangle \text {. }
$$

With a set of operators spanning a complete and orthogonal basis with respect to some symmetry group, these overlaps (coupling constants) give the complete information about symmetry breaking. The interpolating composite operators $O_{i}$ are not normalized on the lattice and consequently the absolute 
values of the coupling constants $a_{i}^{(n)}$ cannot be obtained. However, a ratio of the couplings is a well defined quantity and can be computed as [4]

$$
\frac{a_{i}^{(n)}}{a_{k}^{(n)}}=\frac{\widehat{C}(t)_{i j} u_{j}^{(n)}}{\widehat{C}(t)_{k j} u_{j}^{(n)}} .
$$

Here $\widehat{C}$ is the cross-correlation matrix from ([6) , a sum is implied for the index $j$ on the right-hand side and $u_{j}^{(n)}$ are the eigenvectors obtained from the generalized eigenvalue problem,

$$
\widehat{C}(t)_{i j} u_{j}^{(n)}=\lambda^{(n)}\left(t, t_{0}\right) \widehat{C}\left(t_{0}\right)_{i j} u_{j}^{(n)}
$$

with $t_{0}$ being some normalization point in Euclidean time. The ratio (8) coincides with the ratio of matrix elements (5) with $i \equiv V ; k \equiv T$.

The chiral basis in the quark-antiquark system is a complete one and can be connected to the complete angular momentum basis in the rest frame via the unitary transformation [7]

$$
\left(\begin{array}{l}
\left|(0,1) \oplus(1,0) ; 11^{--}\right\rangle \\
\left|(1 / 2,1 / 2)_{b} ; 11^{--}\right\rangle
\end{array}\right)=U \cdot\left(\begin{array}{c}
\left|1 ;{ }^{3} S_{1}\right\rangle \\
\left|1 ;{ }^{3} D_{1}\right\rangle
\end{array}\right)
$$

with

$$
U=\left(\begin{array}{cc}
\sqrt{\frac{2}{3}} & \sqrt{\frac{1}{3}} \\
\sqrt{\frac{1}{3}} & -\sqrt{\frac{2}{3}}
\end{array}\right) .
$$

Consequently, if we know the mixture of the two allowed chiral representations in a physical state, then applying the unitary transformation (10) we are also able to obtain the angular momentum content of this state in the rest frame. The mixture of the chiral representations in the $\rho$-meson wave function at the resolution scale $\mu$ is given by (8) and (5) and can be measured in lattice simulations.

By this we define what is meant under the angular momentum content of the $\rho$-meson in the rest frame and, consequently, we can answer the question whether or not the spin of the $\rho$-meson is carried by spins of its valence quarks in the rest frame.

\section{Scale dependence of the chiral and angular momentum de- compositions}

The ratio $a_{V}^{(n)} / a_{T}^{(n)}$ as well as a partial wave content of a hadron are not the renormalization group invariant quantities. Hence they manifestly depend on a resolution scale at which we probe the hadron. If we probe the hadron structure on the lattice with the local interpolators, then we study the hadron decomposition at the scale fixed by the lattice spacing $a$. For a reasonably small $a$ this scale is close to the ultraviolet scale. However, we are interested in the hadron content at the infrared scales, where mass is generated. For this purpose we cannot use a large $a$, because matching with the continuum QCD will be lost. Given a fixed, reasonably small lattice spacing $a$ a small resolution scale $\mu \sim 1 / R$ can be achieved by the gauge-invariant smearing of the point-like interpolators. We smear every quark field in spatial directions with the Gaussian profile over the size $R$ in physical units such that $R / a \gg 1$, see Fig. 1. Then even in the continuum limit $a \rightarrow 0$ we probe the hadron content at the resolution scale fixed by $R$. Such a definition of the resolution is similar to the experimental one, where an external probe is sensitive only to quark fields (it is blind to gluonic fields) at a resolution that is determined by the momentum transfer in spatial directions.

We use three different smearing radii $\mathrm{R}$ for the quark fields both in the source and sink. The "narrow" smearing width (index $n$ ) varies between 0.33 and $0.36 \mathrm{fm}$, depending on the set of configurations. The 

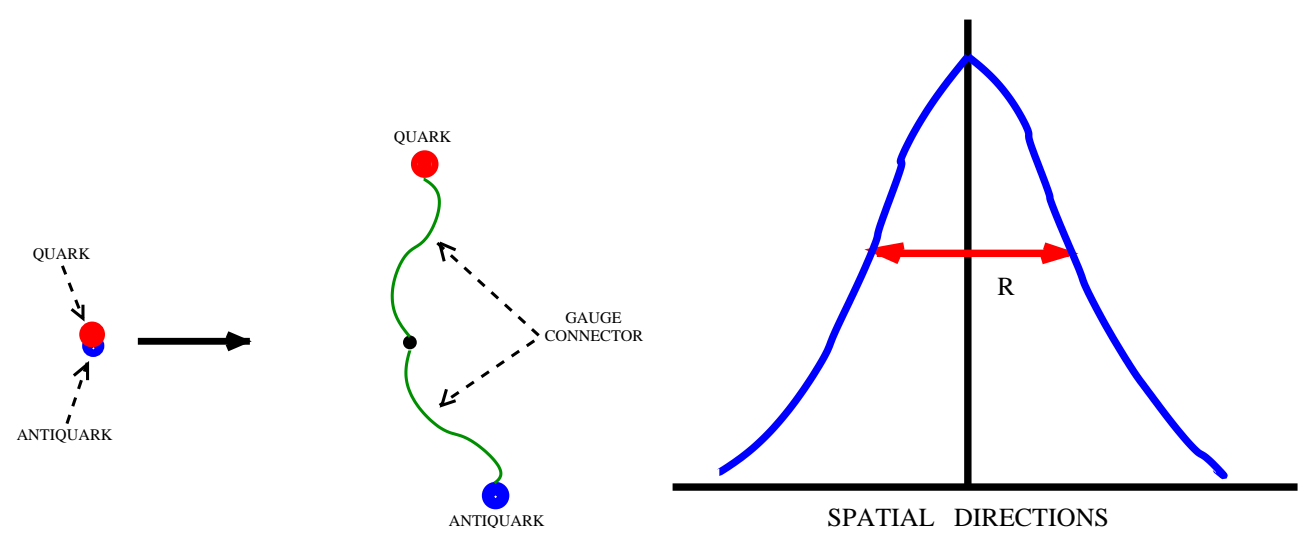

Figure 1: Gauge-invariant smearing and the resolution scale $R$ definition

\begin{tabular}{lccccccc}
\hline \hline Set & $\beta_{L W}$ & $a m_{0}$ & \#conf & $a[\mathrm{fm}]$ & $m_{\pi}[\mathrm{MeV}]$ & $m_{\rho}[\mathrm{MeV}]$ & $m_{\rho^{\prime}}[\mathrm{MeV}]$ \\
\hline A & 4.70 & -0.050 & 200 & $0.1507(17)$ & $526(7)$ & $911(11)$ & $1964(182)$ \\
B1 & 4.65 & -0.060 & 300 & $0.1500(12)$ & $469(5)$ & $870(10)$ & $1676(106)$ \\
B2 & 4.65 & -0.070 & 200 & $0.1406(11)$ & $296(6)$ & $819(18)$ & $1600(181)$ \\
C & 4.58 & -0.077 & 300 & $0.1440(12)$ & $323(5)$ & $795(15)$ & $1580(159)$ \\
\hline \hline
\end{tabular}

Table 1: Specification of the data used here; for the gauge coupling only the leading value $\beta_{L W}$ is given, $m_{0}$ denotes the bare mass parameter of the CI action. Further details on the action, the simulation and the determination of the lattice spacing and the $\pi$ - and $\rho$-masses are found in [13, 14].

"wide" smearing radius (index $w$ ) lies between 0.66 and $0.69 \mathrm{fm}$ and the "ultrawide" one is $0.81-0.85$ $\mathrm{fm}$ (index $u w)$. Hence we can study the hadron structure at resolutions $0.33 \mathrm{fm}-0.85 \mathrm{fm}$.

Consequently we have the following set of the interpolating operators

$$
\begin{array}{ccc}
O_{n}^{V}=\bar{u}_{n} \gamma^{i} d_{n}, & O_{w}^{V}=\bar{u}_{w} \gamma^{i} d_{w}, & O_{u w}^{V}=\bar{u}_{u w} \gamma^{i} d_{u w} \\
O_{n}^{T}=\bar{u}_{n} \gamma^{t} \gamma^{i} d_{n}, & O_{w}^{T}=\bar{u}_{w} \gamma^{t} \gamma^{i} d_{w}, & O_{u w}^{T}=\bar{u}_{u w} \gamma^{t} \gamma^{i} d_{u w}
\end{array}
$$

where $\gamma^{i}$ is one of the spatial Dirac matrices and $\gamma_{t}$ is the $\gamma$-matrix in (Euclidean) time direction.

\section{Lattice details and choices of correlation matrix}

In our study we use Chirally Improved fermions [11] and the Lüscher-Weisz gauge action [12. The lattice size is $16^{3} \times 32$. We use dynamical gauge configurations with two mass-degenerate light quarks. With the lattice spacing $\approx 0.15 \mathrm{fm}$ the spatial volume of the lattice is $\approx 2.4^{3} \mathrm{fm}^{3}$. In our present study

\begin{tabular}{lccc}
\hline \hline Set & $R_{n}[\mathrm{fm}]$ & $R_{w}[\mathrm{fm}]$ & $R_{u w}[\mathrm{fm}]$ \\
\hline A & 0.36 & 0.67 & - \\
B1 & 0.34 & 0.69 & 0.81 \\
B2 & 0.34 & 0.66 & 0.85 \\
C & 0.33 & 0.66 & - \\
\hline \hline
\end{tabular}

Table 2: Specification of the smearing radii $R$. 


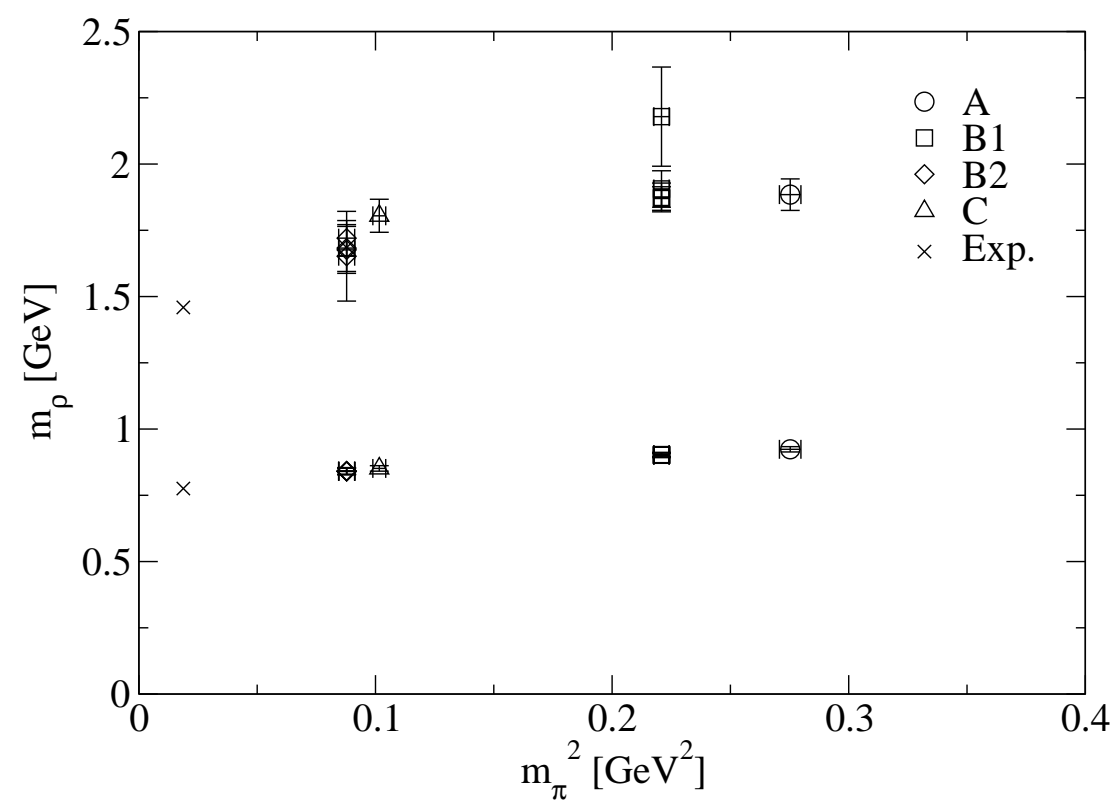

Figure 2: The masses of both $\rho$ and $\rho^{\prime}$ states extracted from different $4 \times 4$ and $6 \times 6$ correlation matrices. The crosses indicate the mass values from experiments.

we limit ourselves to the $\rho$ and $\rho^{\prime}$ states. For details on the simulation we refer the reader to the Table 1 and to [13, 14] .

For the sets $\mathrm{A}$ and $\mathrm{C}$ we construct $4 \times 4$ correlation matrices (i.e., with both vector and pseudotensor interpolators using narrow and wide smearing radii), while for the sets B1 and B2 we study the $6 \times$ 6 correlation matrix (with narrow, wide and ultrawide smearings for both vector and pseudotensor operators) as well as different $4 \times 4$ sub-matrices.

In Fig. 2 we show masses of both the ground state $\rho$-meson and its first excitation $\rho^{\prime}$ extracted from different correlation matrices.

\section{Chiral symmetry breaking and the angular momentum con- tent of $\rho$ and $\rho^{\prime}$ mesons}

The ratio $a_{V} / a_{T}$ of the two allowed chiral representations in the $\rho$ - and $\rho^{\prime}$-mesons versus the resolution $R$ is shown on Fig. 3. The results obtained from different $4 \times 4$ and $6 \times 6$ correlation matrices are consistent with each other. For the ground state $\rho$ we observe strong chiral symmetry breaking practically at all resolutions; only in the deep ultraviolet $R, a \ll 0.3$, where chiral symmetry is unbroken, the tensor "current" decouples from the $\rho$-meson. For the $\rho^{\prime}$ the tensor operator decouples much faster towards the ultraviolet than for the $\rho$-meson. This shows that the wave functions of these two states are significantly different and the chiral symmetry breaking is different in both states. At the scale of $\sim 1$ $\mathrm{fm}$ of the hadron size, i.e., at which the hadron mass is generated, chiral symmetry breaking in the $\rho^{\prime}$ state is less essential than in the ground state.

Given this ratio and the unitary transformation from the chiral basis to the angular momentum basis (10) one can obtain the partial wave content of both states. For the $\rho$ meson it is approximately $0.99\left|{ }^{3} S_{1}\right\rangle-0.1\left|{ }^{3} D_{1}\right\rangle$. Hence the ground state in the infrared is practically a pure ${ }^{3} S_{1}$ state with a tiny admixture of the ${ }^{3} D_{1}$ wave. Consequently in the rest frame the spin of the $\rho$-meson is almost completely carried by spins of its valence quarks. No trace of the "spin crisis" is observed. In this our definition we do not separate contributions of quarks and gluons into hadron spin. In the confining 


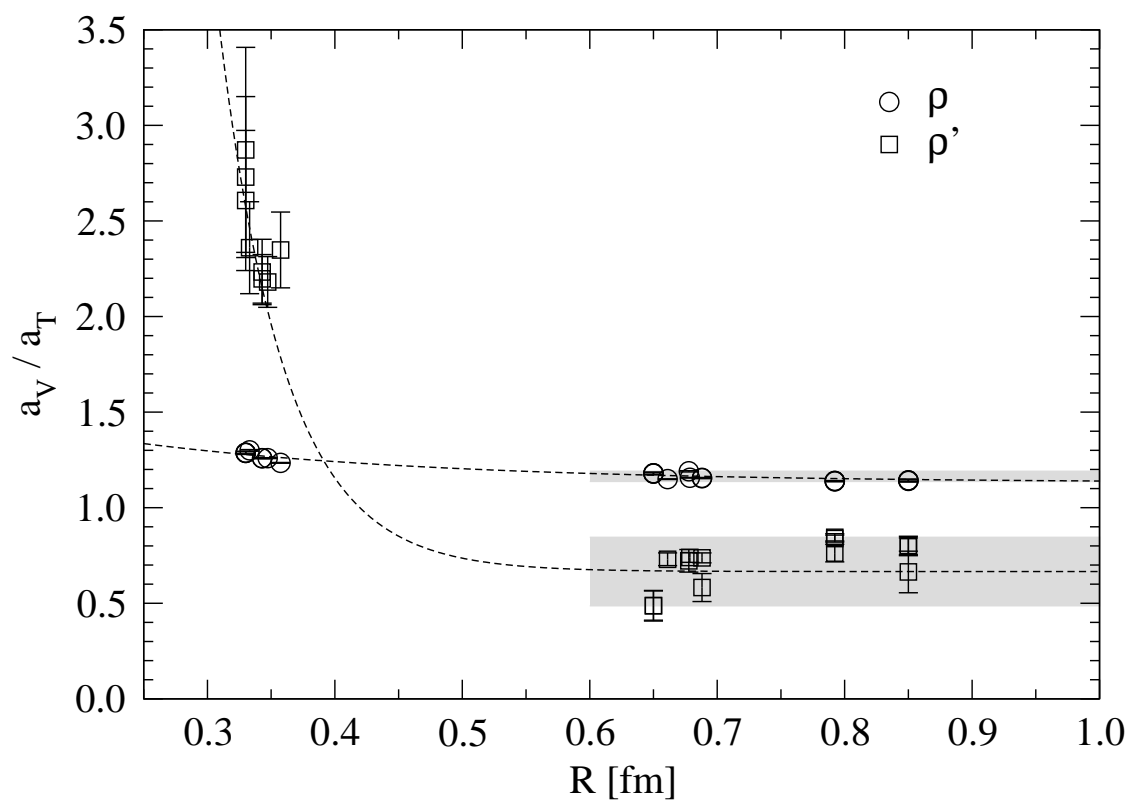

Figure 3: A ratio of the vector to the pseudotensor couplings versus a resolution scale $R$, as extracted from all $4 \times 4$ and $6 \times 6$ correlation matrices. Broken lines are drawn only to guide the eye.

regime, where the gauge invariance and the quark-gluon interaction are of crucial importance, it is not possible to separate both contributions into total spin in a sensible gauge-invariant manner; after all the quark-gluon interaction is a consequence of the gauge invariance. The spin of the $\rho$-meson in the rest frame is carried by spins of its valence quarks dressed by gluons. The gluonic field is important for the angular momentum generation, because it is this field that provides chiral symmetry breaking and that is responsible for most of the hadron mass.

In the excited $\rho$-meson there is a significant contribution of the ${ }^{3} D_{1}$ wave. In the latter case the angular momentum content is between the following two lower and upper bound values. For the lower bound it is $0.88\left|{ }^{3} S_{1}\right\rangle-0.48\left|{ }^{3} D_{1}\right\rangle$ and for the upper bound it is $0.97\left|{ }^{3} S_{1}\right\rangle-0.25\left|{ }^{3} D_{1}\right\rangle$. This once again demonstrates that the first excitation of the $\rho$-meson cannot be considered a pure radial excitation of the ground state $\rho$. Obviously, both radial and orbital degrees of freedom are excited which reflects yet unknown dynamics of confinement and chiral symmetry breaking.

Acknowledgments. We gratefully acknowledge support of the grants P21970-N16 and DK W1203N08 of the Austrian Science Fund FWF and the DFG project SFB/TR-55. The calculations have been performed on the SGI Altix 4700 of the Leibniz-Rechenzentrum Munich and on the local clusters at the University of Graz.

\section{References}

[1] J. Ashman et al. [European Muon Collaboration], Phys. Lett. B 206 (1988) 364; B. Adeva et al. [Spin Muon Collaboration], Phys. Rev. D 58 (1998) 112002.

[2] E. S. Ageev et al. [COMPASS Collaboration], Phys. Lett. B 612 (2005) 154; V. Y. Alexakhin et al. [COMPASS Collaboration], Phys. Lett. B 647 (2007) 8; A. Airapetian et al. [HERMES Collaboration], Phys. Rev. D 75 (2007) 012007.

[3] Ph. Hägler, Phys. Rept. 490 (2010) 49. 
[4] L. Y. Glozman, C. B. Lang and M. Limmer, Phys. Rev. Lett. 103 (2009) 121601; Few Body Syst. 47 (2010) 91.

[5] L. Y. Glozman, C. B. Lang and M. Limmer, Phys. Rev. D 82 (2010) 097501.

[6] L. Y. Glozman, C. B. Lang, M. Limmer, Phys. Lett. B705, 129-133 (2011). arXiv:1106.1010 [hep-ph]].

[7] L. Y. Glozman and A. V. Nefediev, Phys. Rev. D 76 (2007) 096004; Phys. Rev. D 80 (2009) 057901 .

[8] T. D. Cohen and X. D. Ji, Phys. Rev. D 55 (1997) 6870.

[9] L. Y. Glozman, Phys. Lett. B 587, 69 (2004); Phys. Rept. 444, 1 (2007)

[10] C. Michael, Nucl. Phys. B 259 (1985) 58; M. Lüscher and U. Wolff, Nucl. Phys. B 339, 222 (1990).

[11] C. Gattringer, Phys. Rev. D 63 (2001) 114501; C. Gattringer, I. Hip, and C. B. Lang, Nucl. Phys. B 597 (2001) 451 (2001).

[12] M. Lüscher and P. Weisz, Commun. Math. Phys. 9759 (1985); [Erratum-ibid. 98 (1985) 433].

[13] C. Gattringer, C. Hagen, C. B. Lang, M. Limmer, D. Mohler and A. Schäfer, Phys. Rev. D 79 (2009) 054501.

[14] G. P. Engel, C. B. Lang, M. Limmer, D. Mohler and A. Schäfer, Phys. Rev. D 82 (2010) 034505. 